\title{
Abnormal Continuous Flow in Giant \\ Intracranial Aneurysm Detected by \\ Transcranial Doppler Sonography
}

\author{
-Case Report-
}

\author{
Yasushi Kurokawa, Tomomi OKAMURA, Seisho ABIKo \\ and Kohsaku WATANABE
}

Department of Neurosurgery, Ube Industries Central Hospital, Ube, Yamaguchi

\begin{abstract}
Transcranial Doppler sonography demonstrated a huge, non-thrombosed intracranial aneurysm in a 62-year-old male preoperatively as abnormal continuous flow, which disappeared after aneurysmal neck clipping. Transcranial Doppler sonography is very useful to assess hemodynamics in the parent artery and the dome of the aneurysm.
\end{abstract}

Key words: giant aneurysm, transcranial Doppler sonography, flow velocity, middle cerebral artery

\section{Introduction}

Transcranial Doppler sonography (TCD) is very useful for the non-invasive evaluation of intracranial hemodynamics in many cerebrovascular diseases, and definite and diagnostic findings can be obtained. ${ }^{1,2,4,6,7,9-11)}$ However, the hemodynamic abnormalities caused by intra-aneurysmal blood flow are unclear, and the identification of intracranial aneurysms is uncertain.

We present a case of successfully clipped huge intracranial aneurysm, and discuss the usefulness of TCD to detect the intra-aneurysmal blood flow and to evaluate the hemodynamic changes caused by aneurysm clipping.

\section{Case Report}

A 62-year-old male was admitted with mild headache persisting for several months. Physical examination revealed left divergent nystagmus, which he had had since childhood. Postcontrast computed tomographic (CT) scans showed a round, homogeneously enhanced lesion in the left paraclinoid area, extending upward (Fig. 1). Left carotid angiograms

Received January 6, 1992; Accepted May 21, 1992

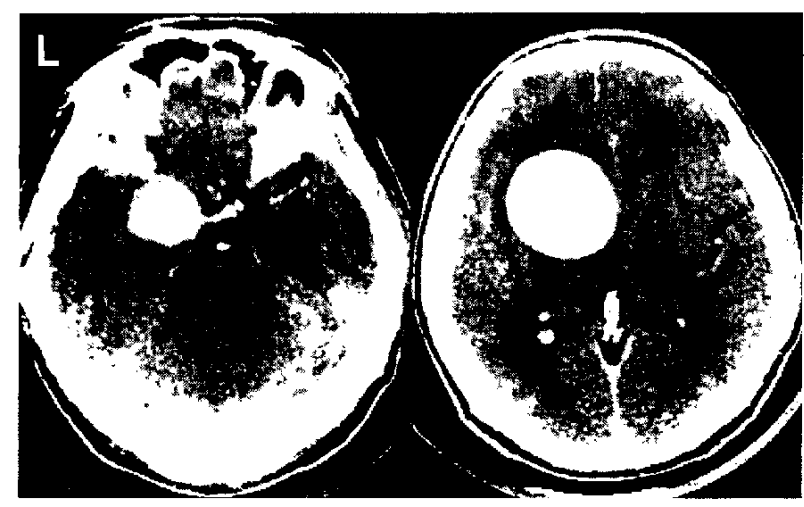

Fig. 1 Postcontrast CT scans, showing a round, homogeneously enhanced lesion, extending upward from the paraclinoid area.

demonstrated a huge aneurysm originating from the left middle cerebral artery (MCA), with the distal MCA branches poorly visualized (Fig. 2). The aneurysmal neck was located at the distal end of the $\mathbf{M}_{1}$ segment. The aneurysm was $55 \mathrm{~mm}$ in diameter and showed neither apparent calcification nor intraaneurysmal thrombosis. Regional cerebral blood flow (rCBF) in the MCA region measured by cold $\mathrm{Xe} C \mathrm{~T}$ was $41.4 \mathrm{ml} / 100 \mathrm{gm} / \mathrm{min}$ on the right and 


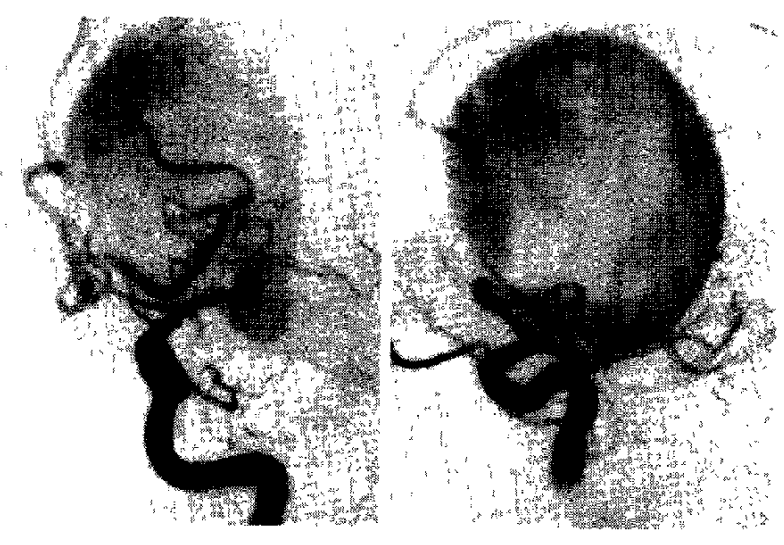

Fig. 2 Left internal carotid angiograms, anteroposterior (left) and lateral (right) views, showing a huge, non-thrombosed aneurysm originating from the left MCA, with the poorly visualized distal MCA branches.
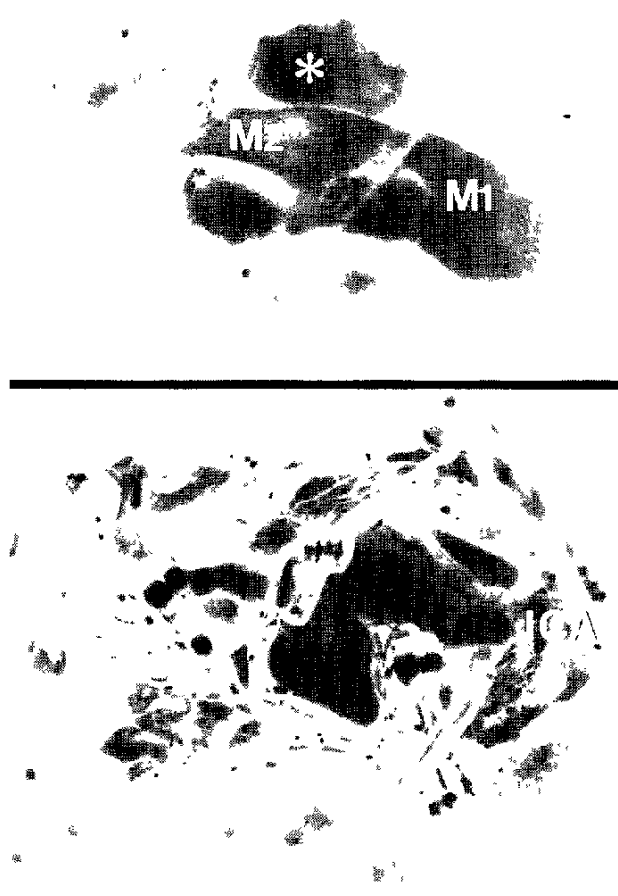

Fig. 3 Operative views, before (upper) and after (lower) clipping of the aneurysmal neck and $\mathbf{M}_{1}$ formation by a tandem clipping technique. *: aneurysmal dome, ICA: left internal carotid artery, $M_{1}$ and $M_{2}: M_{1}$ and $M_{2}$ portions of the left MCA.

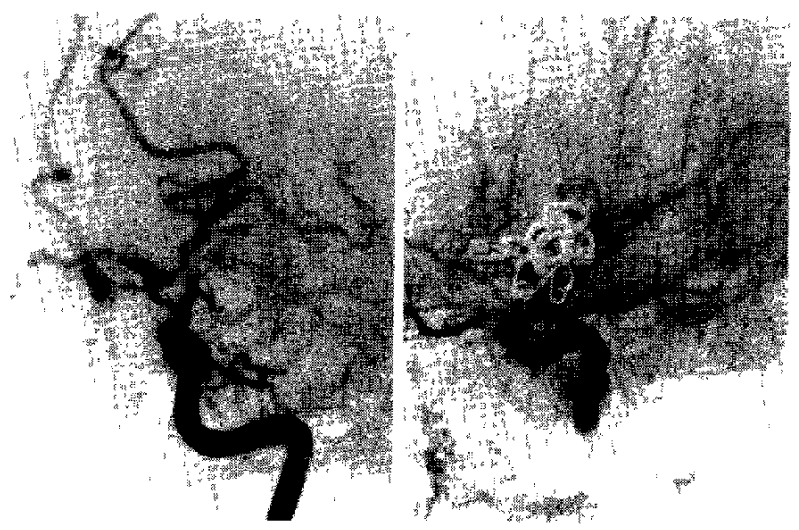

Fig. 4 Postoperative left internal carotid angiograms, anteroposterior (left) and lateral (right) views, showing successful clipping of the aneurysm and $\mathrm{M}_{1}$ formation, although blood circulation to the distal MCA branches is delayed.

\section{$23.7 \mathrm{ml} / 100 \mathrm{gm} / \mathrm{min}$ on the left.}

A left frontotemporal craniotomy with him in the supine position was performed with continuous monitoring of the $\mathrm{rCBF}$. A wide dissection of the left Sylvian fissure exposed the aneurysm, internal carotid artery, anterior cerebral artery, and the $\mathbf{M}_{2}$ portion of the MCA (Fig. 3 upper). The aneurysmal wall was punctured and the blood content aspirated after temporary clipping of the internal carotid artery, anterior cerebral artery, and the $\mathrm{M}_{2}$ portion of the MCA. The aneurysmal dome shrank, allowing easy neck clipping and $\mathrm{M}_{1}$ formation using three fenestrated Sugita clips and a curved clip (Fig. 3 lower). The aneurysmal dome did not re-expand after removal of the temporary clips.

Postoperative angiograms verified the successful clipping of the aneurysm and $\mathbf{M}_{1}$ formation, but blood circulation to the distal MCA branches was delayed (Fig. 4). rCBF in the left MCA region was $24.5 \mathrm{ml} / 100 \mathrm{gm} / \mathrm{min}$. Postoperatively, he recovered uneventfully except for a transient mild dyscalculia. He was discharged 1 month later.

TCD used pulsed wave, range-gated, directional 2$\mathrm{MHz}$ Doppler equipment with acoustical focusing and real-time spectrum analysis. The probe was placed on the temporal region and flow velocities (FVs) and pulsatility index (PI) recorded. Preoperatively, signals from both the aneurysmal neck and the inside of the aneurysm were recorded. The FV of the aneurysmal neck recorded at $40 \mathrm{~mm}$ depth showed reduced pulsatility (Fig. 5A), and inside the aneurysm recorded at $50-65 \mathrm{~mm}$ depth was not pulsative but continuous (Fig. 5B, C). The intra- 


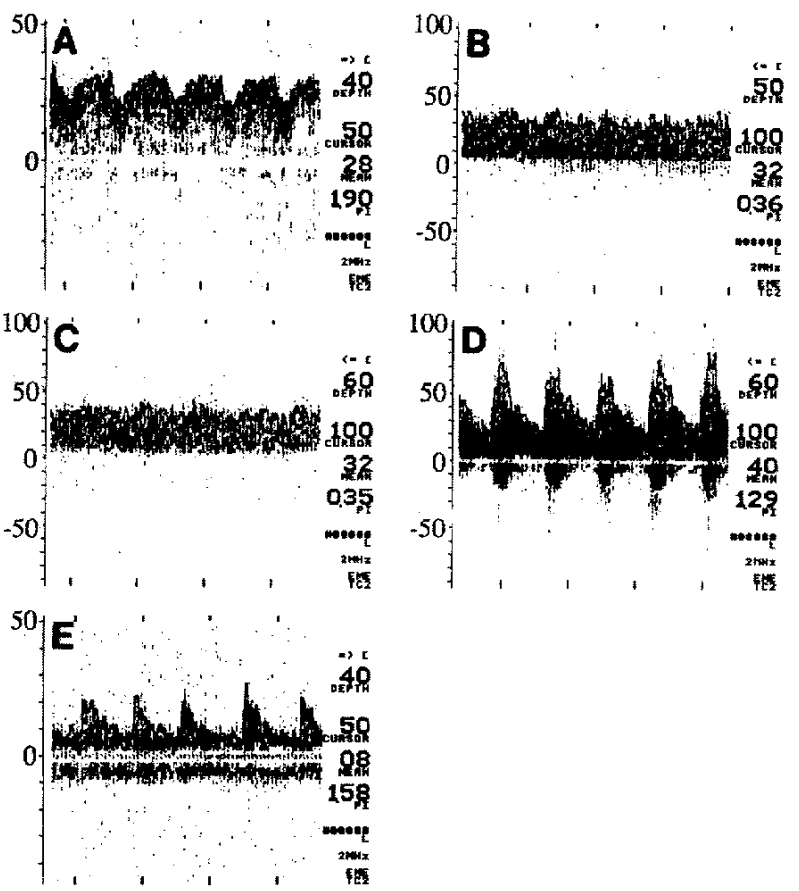

Fig. 5 TCD findings from left anterior circulation. A: Recordings from aneurysmal dome near the neck, showing reduced pulsatility. B, C: Recordings from the aneurysmal dome center, showing continuous and reversed flow with low FV and low PI. D: Recordings from the bifurcation of the left internal carotid artery, showing bidirectional flow with high PI. E: Postoperative recordings from the distal end of the MCA, showing normal flow direction, although the $\mathrm{FV}$ remained low.

aneurysmal flow direction was reversed between 40 and $50 \mathrm{~mm}$ depth. The recordings at the left internal carotid artery bifurcation showed bidirectional flow, low FV and high PI (Fig. 5D). The recordings from the right side were almost normal. After neck clipping and $\mathbf{M}_{1}$ formation, the abnormal continuous flow completely disappeared, although the FV at the distal end of the MCA remained low (Fig. 5E).

\section{Discussion}

We recorded TCD in a case of partially thrombosed giant aneurysm, but found no abnormal findings from the aneurysm. ${ }^{5)}$ However, we could precisely evaluate the hemodynamic changes before and after operation. In the present case, changes in the FVs and the PI were recorded after neck clipping and $M_{1}$ formation. Therefore, the hemodynamic state of the perianeurysmal area can always be evaluated. Huge, non-thrombosed intracranial aneurysms can be detected because TCD shows abnormal FVs and PI. The detection of aneurysms using Doppler sonography is not well known, ${ }^{8)}$ so we cannot offer definitive diagnostic Doppler findings for aneurysm.

Interpretation of Doppler findings from aneurysms should take account of the intraaneurysmal conditions, including thrombosis, blood volume, and flow direction. If the aneurysm is very small, it is impossible to detect the aneurysm directly. Also, a giant aneurysm that is thrombosed will cause no abnormal Doppler sonography findings. In contrast, abnormal findings from nonthrombosed giant aneurysms are easily detected. In our case, the rich and rapid flow in the aneurysmal dome was accurately detected as continuous flow. Nornes et $a l^{8)}$ reported the intraoperative evaluation of cerebral hemodynamics using directional Doppler sonography. This technique, used for localization and targeting the operative approach, could detect the aneurysm by the characteristic intra-aneurysmal flow pattern, due to a "whirlpool-like motion" in the aneurysmal dome. In addition, Ferguson ${ }^{3)}$ and German and Black, ${ }^{4}$ in studies of intra-aneurysmal hemodynamics, concluded that the flow-through time in an aneurysm is very low and flow is highly irregular and turbulent. In our case, the "whirlpoollike motion"' may have appeared as the abnormal continuous flow. Doppler signals obtained from near the aneurysmal neck (at $40 \mathrm{~mm}$ depth in the left temporal region) showed reduced pulsatility in the aneurysm. Signals obtained from the aneurysmal center (at 50-65 mm depth) showed reversed and continuous flow. These changes in intra-aneurysmal FVs

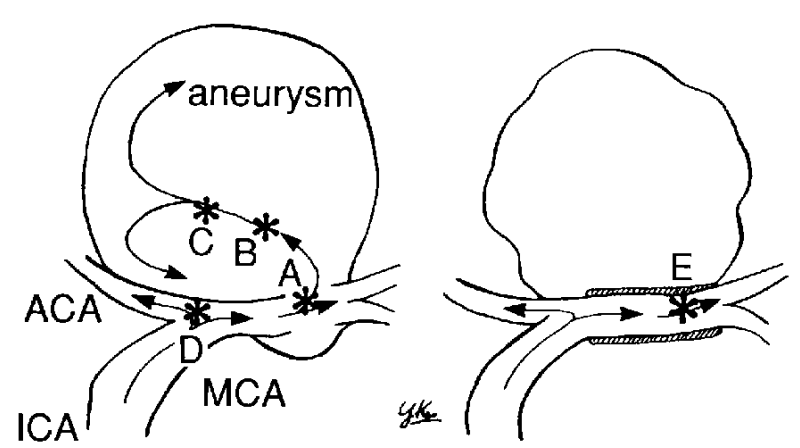

Fig. 6 Schematic drawing of the perianeurysmal hemodynamics before (left) and after (right) clipping and $\mathrm{M}_{1}$ formation. *: foci of the TCD recordings in Fig. 5, ACA: left anterior cerebral artery, ICA: left internal carotid artery. 
reflect the intra-aneurysmal whirlpool-like blood flow (Fig. 6).

The intra-aneurysmal hemodynamics will differ in individual cases, so abnormal continuous flow in the aneurysmal dome cannot always be detected. However, there is a good possibility that TCD can detect abnormal flow in the dome of non-thrombosed giant aneurysms. This procedure will be important in the non-invasive study of intra-aneurysmal hemodynamics.

\section{Acknowledgment}

This study was presented in part at the 48th Annual Meeting of the Japan Neurosurgical Society, held in September, 1989, Morioka, Japan.

\section{References}

1) Aaslid $R$, Huber $P$, Nornes $H$ : Evaluation of cerebrovascular spasm with transcranial Doppler ultrasound. J Neurosurg 60: 37-41, 1984

2) Aaslid R, Markwalder TM, Nornes H: Noninvasive transcranial Doppler ultrasound recording of flow velocity in basal arteries. $J$ Neurosurg 57: 769-774, 1982

3) Ferguson GG: Turbulence in human intracranial saccular aneurysms. $J$ Neurosurg 33: 485-497, 1970

4) German WJ, Black SPW: Intra-aneurysmal hemodynamics: Turbulence. Trans Amer Neurol Ass 79: 163-165, 1954

5) Kurokawa Y, Okamura T, Watanabe K, Abiko S,
Katoh S: Assessment of hemodynamic state of giant aneurysms by transcranial Doppler sonography, in: Abstract of the 48th Annual Meeting of the Japan Neurosurgical Society. 1989, p 338 (in Japanese)

6) Lindegaard KF, Bakke SJ, Aaslid R, Nornes H: Doppler diagnosis of intracranial artery occlusive disorders. I Neurol Neurosurg Psychiatry 49: 510518,1986

7) Lindegaard K-F, Bakke SJ, Grolimund P, Aaslid R, Huber $P$, Nornes $H$ : Assessment of intracranial hemodynamics in carotid artery disease by transcranial Doppler ultrasound. $J$ Neurosurg 63: 890-898, 1985

8) Nornes H, Grip A, Wikeby P: Intraoperative evaluation of cerebral hemodynamics using directional Doppler technique. $J$ Neurosurg 50: 570-577, 1979

9) Seiler RW, Grolimund P, Aaslid R, Huber P, Nornes $\mathrm{H}$ : Cerebral vasospasm evaluated by transcranial ultrasound correlated with clinical grade and CTvisualized subarachnoid hemorrhage. $J$ Neurosurg 64: 594-600, 1986

10) Spencer MP, Whisler D: Transorbital Doppler diagnosis of intracranial arterial stenosis. Stroke 17: 916-921, 1986

11) Wechsler LR, Ropper AH, Kistler JP: Transcranial Doppler in cerebrovascular disease. Stroke 17: 905912, 1986

Address reprint requests to: Y. Kurokawa, M.D., Department of Neurosurgery, Ube Industries Central Hospital, 750 Nishikiwa, Ube, Yamaguchi 755-01, Japan. 